\title{
AP Association for Proportional Fairness in Multi-rate WLANs
}

\author{
Wei Li, Student Member, IEEE, Shengling Wang, Member, IEEE, Yong Cui, Member, IEEE, \\ Xiuzhen Cheng, Senior Member, IEEE, Ran Xin, Mznah A. Al-Rodhaan, Abdullah Al-Dhelaan
}

\begin{abstract}
In this study, we investigate the problem of achieving proportional fairness via AP association in multi-rate WLANs. This problem is formulated as a non-linear programming with an objective function of maximizing the total user bandwidth utilities in the whole network. Such a formulation jointly considers fairness and AP selection. We first propose a centralized algorithm NLAO-PF to derive the user-AP association via relaxation. Since the relaxation may cause a large integrality gap, a compensation function is introduced to ensure that our algorithm can achieve at least half of the optimal in the worst-case. This algorithm is assumed to be adopted periodically for resource management. To handle the case of dynamic user membership, we propose a distributed heuristic BPF based on a novel performance revenue function, which provides an AP selection criterion for newcomers. When an existing user leaves the network, the transmission times of other users associated with the same AP can be redistributed easily based on NLAO-PF. Extensive simulation study has been performed to validate our design and to compare the performance of our algorithms with those of the state-of-the-art.
\end{abstract}

Index Terms-AP association; bandwidth allocation; multi-rate WLANs; proportional fairness.

\section{INTRODUCTION}

B Y default, each user in an IEEE 802.11 WLAN associates with the AP that has the largest received signal strength indicator (RSSI). As typically users are not uniformly distributed among all APs, RSSI based approach may overload some APs while leave others to carry very light load or even to be idle. This load unbalancing could result in unfair bandwidth allocation. Although the network is supposed to serve fairly at high performance, fairness and efficiency are often in conflict with each other. With the development of multi-rate WLANs, this problem has become even more challenging, as users with different bit rates intend to share the same WLAN.

It is well-known that the popular 802.11 MAC protocol provides equal long-term transmission opportunities to all

Wei Li and Xiuzhen Cheng are with Department of Computer Science, The George Washington University, Washington DC, USA. Email: \{weili, cheng\} @gwu.edu.

Shengling Wang is with the Institute of Computing Technology, Chinese Academy of Sciences, Beijing, China. Email: wangshengling@ict.ac.cn.

Yong Cui is with Department of Computer Science, Tsinghua University, Beijing, P. R. China. Email: cuiyong@tsinghua.edu.cn.

Ran Xin is with Department of Computer Science, Illinois Institute of Technology, Chicago, USA. Email: rxin@iit.edu.

Mznah A. Al-Rodhaan and Abdullah Al-Dhelaan are with College of Computer and Information Sciences, King Saud University, Riyadh, Saudi Arabia. Email: \{rodhaan, dhelaan\}@ksu.edu.sa.

This work has been supported by the National Science Foundation of the US (CNS-0831852), the NPST program by King Saud University (Project No. 10-INF1184-02), and the National Natural Science Foundation of China (Grant No. 61272475 and 61003225). users associated with the same AP. Therefore users with the same frame size and same transmission rate can achieve equal throughput (i.e. throughput-based fairness). However, in multirate WLANs, throughput-based fairness requires that users with lower bit rates occupy the channel for longer times than those with higher bit rates, drastically reducing the network throughput [1], [2]. To overcome this problem, time-based fairness is proposed such that each user can obtain an equal share of channel occupancy time. Recent research [1], [3] has shown that time-based fairness outperforms throughput-based fairness in multi-rate WLANs.

There exist other fairness criteria that are widely adopted in network resource assignment. Max-min fairness distributes resources as equally as possible among users [4]-[7]; proportional fairness, on the other hand, allocates bandwidth to users in proportion to their bit rates to maximize the sum of the bandwidth utilities of all users [8]-[10]. Proportional fairness has been utilized to effectively exploit the tradeoff between fairness and network performance [8], [11]. It is argued [12] that within a single saturated AP, throughput-based fairness and max-min fairness are equivalent; moreover, if all users have the same priority level, time-based fairness and proportional fairness are also equivalent.

Fairness and AP association should be jointly considered for resource management in multi-rate WLANs but existing research mainly focuses on the joint study of max-min fairness and AP association. In this paper, we investigate the problem of achieving proportional fairness via AP association for performance enhancement. To achieve this goal, we formulate the problem to a non-linear programming (NLP) with an objective function of maximizing the total user bandwidth utilities in the whole network, and propose a centralized algorithm termed Non-Linear Approximation Optimization for Proportional Fairness (NLAO-PF) to solve it. Since the objective function of our NLP is nonlinear and the AP association is a 0-1 integer programming problem, NLP is NP-hard [13]. Therefore NLAO-PF is decomposed into four steps to simplify the issue and improve the degree of approximation. By introducing a compensation function to the objective function of NLP, the total utility of the bandwidth allocation via NLAO$\mathbf{P F}$ is proved to be at least half of the optimal in the worst case. In real world applications, NLAO-PF can be adopted periodically to achieve proportional fairness. To handle the case of dynamic user membership, we propose a distributed heuristic termed Best Performance First (BPF) based on a novel performance revenue function, which provides an AP selection criterion for the newly-arriving users. When an 
existing user leaves the network, the transmission times of other users associated with the same AP can be redistributed easily based on NLAO-PF. Our comparison-based simulation study indicates that both NLAO-PF and BPF perform well when the users are distributed randomly and uniformly in the whole network. Moreover, the superiority of our algorithms is even higher compared to the most relevant ones when users are distributed in a hot spot area.

The rest of the paper is organized as follows. The most related work is discussed in Section II. The fairness criterion and our network model are introduced in Section III. The two algorithms are detailed in Sections IV and V. After presenting the evaluation results in Section VI, we conclude this paper in Section VII.

\section{RELATED WORK}

Achieving fairness within a single AP via optimizing the media access procedure has been extensively studied. The fairness of CSMA/CA is analyzed by Jian and Chen in [14]. This work also proposes a rate control protocol called "Proportional Increase Synchronized Multiplicative Decrease" to achieve fair bandwidth allocation. The short-term and longterm fairness of the 802.11 DCF procedure are investigated in [15] by employing the conditional probabilities of the number of inter-transmissions. A technique to estimate the fair rate from passive traffic measurements of a video application is also proposed in [15]. In [1], [16]-[18], different media access methodologies are investigated to optimize the MAC parameters for fairness. In [19], a CSMA/CA MAC protocol without adopting the exponential back-off procedure is proposed to optimize the throughput when achieving time-based fairness by adaptively determining the contention window size based on the transmission opportunities.

AP association is another fundamental problem in wireless networks to enhance the performance. Ekici and Yongacoglu [20] propose a distributed AP selection scheme, in which a user associates with an AP that provides the best performance in terms of congestion relief by considering the bit rate as well as the number of users accommodated by the AP. Abusubaih and Wolisz [21] present a centralized optimal association policy for multi-rate IEEE 802.11 WLANs. Their policy is based on the cell status information and can facilitate information exchange between APs. Yen et al. [22] model AP selection under the framework of game theory, where the sole goal of each wireless station is to maximize its achievable throughput, by considering both the number of wireless stations that associate with the same AP and the set of link rates these wireless stations possess. Keranidis et al. [23] make the AP selection for each user in a distributed way to maximize per-user total throughput, which is defined to be the sum of the throughputs on the uplink and the downlink.

None of the works mentioned above jointly considers fairness and AP association. Achieving throughput-based maxmin fairness via AP association has been studied in [6], [24][26]. Gong et al. [24] formulate the AP selection problem in wireless mesh networks as a nonlinear optimization programming, and apply a weighting parameter to obtain a tradeoff between the total throughput and the max-min fairness. In [25], a two-stage smart association control protocol is proposed. In the bandwidth allocation stage, APs collaboratively determine the number of devices they are going to associate for maxmin fairness; while in the AP association stage, devices are assigned to APs for throughput maximization. Bejerano et al. [6] and $\mathrm{Xu}$ et al. [26] demonstrate the strong correlation between throughput-based max-min fairness and min-max load balancing, and achieve the max-min fair bandwidth allocation via AP association.

Throughput based max-min fairness suffers from a low network throughput in multi-rate WLANs [8], [11]. Proportional fairness, on the other hand, can effectively investigate the tradeoff between fairness and network throughput [8], [11]. Li et al. [11] formulate a non-linear programming to achieve optimal proportional fairness in a network of APs, and propose two approximate AP selection schemes, cvapPF and nlapPF, for periodic off-line optimization. Both cvapPF and nlapPF rely on relaxation and rounding to obtain an integral user-AP association. $\mathrm{Bu}$ et al. [13] study the proportional fairness problem in $3 \mathrm{G}$ wireless data networks. This study is particularly suitable for $3 \mathrm{G}$ data networks and therefore is not applicable to multi-rate WLANs. Koukoutsidis and Siris [27] propose a branch-and-bound algorithm to investigate the network throughput under the max-min fairness and proportional fairness. But the time complexity of this algorithm is inversely proportional to the allowable relative error from the optimal solution, resulting in an unbounded performance in the worst case. Xie et al. [28] formulate the problem of AP association control over vehicular networks as a convex programming in the offline setting, and design a dynamic weight-based online algorithm to achieve proportional fairness. Li et al. [29] jointly consider AP association and power control, establish the relationship between the network utility and the AP utility according to proportional fairness, and then devise a centralized heuristic approach to optimize the network utility by increasing the average and decreasing the variance of the AP utility.

In this paper, we propose two algorithms that jointly consider AP association and fair bandwidth allocation. Our centralized problem formulation is motivated by the non-linear programming in [11] but we adopt a completely different approach to relax the variables in our approximation algorithm design. By introducing a compensation function to the objective function to narrow down the gap caused by relaxation, we achieve an approximate solution that is at least half of the optimal in the worst-case. Our second algorithm is distributed, which selects an AP for a newly-arriving user based on a novel performance revenue function to achieve proportional fairness. Note that our centralized and distributed algorithms can be combined to significantly improve the performance of dynamic networks where users come and leave by their own free will.

\section{Proportional Fairness and Network Model}

In this section, we first briefly introduce the formal definition of proportional fairness; then we detail our network model and problem formulation. 


\section{A. Proportional Fairness}

To fairly assign bandwidths to users while guaranteeing the network performance in multi-rate WLANs, we adopt proportional fairness, a fairness criterion that was proposed by F. P. Kelly [8]. According to [8], proportional fairness can be formally defined as follows. Let $b_{i}$ be the effective bandwidth of user $i$ and $F\left(b_{i}\right)$ be the corresponding utility function. $A$ vector of bandwidth assignment $\left\{b_{1}, b_{2}, \ldots, b_{M}\right\}$, with $M$ being the number of users in the network, is proportionally fair if it is feasible and if for any other feasible vector $\left\{b_{1}^{*}, b_{2}^{*}, \cdots, b_{M}^{*}\right\}$, the aggregate of proportional changes is either zero or negative, i.e.,

$$
\sum_{i=1}^{M} \frac{b_{i}^{*}-b_{i}}{b_{i}} \leq 0 .
$$

Consider a small feasible perturbation $b_{i} \rightarrow b_{i}+\delta b_{i}$, which increases the utility function $F\left(b_{i}\right)$ providing that

$$
\sum_{i=1}^{M} F^{\prime}\left(b_{i}\right) \delta b_{i}>0 .
$$

From the definition of proportional fairness, we have

$$
\sum_{i=1}^{M} \frac{\delta b_{i}}{b_{i}}>0,
$$

which can be rewritten as

$$
\sum_{i=1}^{M}\left(\log \left(b_{i}\right)\right)^{\prime} \delta b_{i}>0 .
$$

Thus it follows that the above proportionally fair bandwidth allocation can be represented by a local maximum of the logarithmic utility function. Since the logarithmic function is differentiable and strictly concave, it has only one maximum and therefore the local maximum is also the global maximum [8]. Accordingly, the objective of a proportionally fair bandwidth allocation can be expressed by

$$
\max \sum_{i=1}^{M} \log \left(b_{i}\right) \text {. }
$$

To quantitatively evaluate the fairness degree of our bandwidth allocation, we adopt Jain's Fairness Index [30], which states that if a system allocates resources (bandwidths in our case) to $M$ users, with the ith user receiving an allocation $b_{i}$, the fairness index of the system is defined to be

$$
J=\frac{\left(\sum_{i=1}^{M} b_{i}\right)^{2}}{M \sum_{i=1}^{M} b_{i}^{2}}, \text { where } b_{i} \geq 0 .
$$

This index measures the "equality" of users' resource allocation $\left\{b_{1}, b_{2}, \ldots, b_{M}\right\}$. If all users obtain the same amount of the bandwidth, i.e., all $b_{i}$ 's are equal, the fairness index is 1 and the system is $100 \%$ fair. As the disparity increases, fairness decreases. An allocation scheme which favors only a few users has a fairness index close to 0 .

\section{B. Network Model}

Our network topology models an IEEE 802.11 based multirate WLAN that consists of multiple APs operating at the same channel. Each AP has the same limited coverage area and serves users in its area. Overlapping coverage areas of adjacent APs may exist. The union of the coverage areas of all APs forms the network coverage area. We assume that each AP transmits messages with the same power as defined by IEEE 802.11. We further assume that each user is covered by at least one AP, and each AP has at least one associated user. The notations and definitions to be utilized are summarized in TABLE I.

TABLE I

NOTATIONS

\begin{tabular}{|c|l|}
\hline Symbol & \multicolumn{1}{c|}{ Semantics } \\
\hline$A$ & The set of all access points (APs) \\
\hline$A_{i}$ & The set of APs associated with user $i$ \\
\hline$N$ & $N=|A|$, the number of APs \\
\hline$U$ & The set of all users \\
\hline$M$ & $M=|U|$, the number of users \\
\hline$\gamma_{i j}$ & The SINR of the link from AP $j$ to user $i$ \\
\hline$g_{i j}$ & The channel gain from AP $j$ to user $i$ \\
\hline$p_{j}$ & The transmit power of AP $\mathrm{j}$ \\
\hline$N_{0}$ & The receiver noise power \\
\hline$w_{i}$ & The weight (priority) of user $i, w_{i}>0$ \\
\hline$b_{i}$ & The effective bandwidth allocated to user $i$ \\
\hline$r_{i j}$ & The bit rate between user $i$ and AP $j$ \\
\hline$x_{i j}$ & The association coefficient between user $i$ and AP $j$ \\
\hline$X$ & The 0-1 user-AP association matrix $\left\{x_{i j}\right\}$ \\
\hline$t_{i j}$ & The effective transmission time between user $i$ and AP $j$ \\
\hline$T$ & The transmission time allocation matrix $\left\{t_{i j}\right\}$ \\
\hline
\end{tabular}

As we have known, a user in an overlapping coverage area is typically serviced by one AP and interfered with all other APs. The effective bit rate of a user in an 802.11 network is determined by the experienced SINR of the user. More precisely, let $\gamma_{i j}$ denote the SINR of user $i$ when associated with AP $j$. We have,

$$
\gamma_{i j}=\frac{g_{i j} p_{j}}{\sum_{k \in \mathbf{A}_{\mathbf{i}} \cap k \neq j} g_{i k} p_{k}+N_{0}},
$$

where $g_{i j}$ is the channel gain from $\mathrm{AP} j$ to user $i, p_{j}$ is the transmit power of $\mathrm{AP} j, N_{0}$ is the additive Gaussian white noise, and $\mathbf{A}_{\mathbf{i}}$ is the set of APs whose transmissions interfere with user $i$. Note that here we choose to focus on downlink because the data transmissions from the APs represent the dominate traffic for many real-world applications such as social networks [31], [32]. The relationship between the effective bit rates and the SINR ranges in an 802.11 network is shown in TABLE II [33], [34].

TABLE II

The Relationship BETWEen SINRS AND EFFECTIVE BIT RATES IN IEEE 802.11 STANDARD

\begin{tabular}{|c|c|c|c|c|c|c|c|c|}
\hline$\gamma_{i j}(\mathrm{~dB})$ & $6-7.8$ & $7.8-9$ & $9-10.8$ & $10.8-17$ & $17-18.8$ & $18.8-24$ & $24-24.6$ & $24.6-$ \\
\hline$r_{i j}(\mathrm{Mbps})$ & 6 & 9 & 12 & 18 & 24 & 36 & 48 & 54 \\
\hline
\end{tabular}

It is assumed that the network is saturated such that all APs are busy all the time to send data to users. A unit of time, in 
which the network is stable, with no new user joins and no current user leaves, is to be considered. This means that under our consideration the total transmission time of an AP is equal to 1. Each AP assigns fractional transmission times to users in accordance with proportional fairness. A user is allowed to choose one and only one AP within a unit time.

We formulate the problem of AP association based on proportional fairness as a non-linear programming. Our goal is to construct an assignment of users to APs in a proportional manner; i.e., the assignment allocates each user a sufficient amount of bandwidth without unduly restricting the amount of bandwidth available to others. In our system model, the resources at all APs are considered as a whole when allocating bandwidth fairly to users. With this network-wide fairness objective, load balancing is automatically taken into account. Since the effective bandwidth of user $i$ is $b_{i}=\sum_{j=1}^{N} x_{i j} t_{i j} r_{i j}$, we obtain the following optimization formulation:

$$
\begin{aligned}
\max & \sum_{i=1}^{M} w_{i} \log \left(\sum_{j=1}^{N} x_{i j} t_{i j} r_{i j}\right) \\
\text { s.t. } & \sum_{j=1}^{N} x_{i j}=1,1 \leq i \leq M, \\
& \sum_{i=1}^{M} x_{i j} t_{i j}=1,1 \leq j \leq N, \\
& x_{i j} \in\{0,1\}, 1 \leq i \leq M, 1 \leq j \leq N, \\
& t_{i j} \in[0,1], 1 \leq i \leq M, 1 \leq j \leq N .
\end{aligned}
$$

Eq. (2) is referred as a Non-linear Programming problem (NLP). Note that our objective function (2a) considers the weights of the users, which reflects their priorities in a real network. The constraint (2b) indicates that each user can associate with one and only one AP; the constraint (2c) requires that the total transmission time of each AP $j$ is equal to 1 ; the constraint (2d) assures that $x_{i j}$ is a binary variable that is equal to 1 if and only if user $i$ associates to AP $j$; and the constraint (2e) specifies the range of the variable $t_{i j}$. We can prove that NLP is NP-hard by slightly adapting the reduction procedure proposed in [13]. Note that this problem formulation is motivated by [11] but our approach to solving the problem via relaxation, as elaborated in Section $\mathrm{IV}$, is fundamentally different and completely novel. Also note that the joint problem of AP association and bandwidth allocation expressed by (2) is formulated based on time-based proportional fairness, given the bit rates between users and APs.

\section{The NLAO-PF ALGORITHM}

\begin{tabular}{l}
\hline Algorithm 1 NLAO-PF \\
\hline 1: Obtain $\left\{t_{i j}^{\prime}\right\}$ by solving r-NLP. \\
2: Get the fractional solution $\left\{x_{i j}^{\prime}\right\}$ by solving c-NLP. \\
3: Get the integral solution $\left\{x_{i j}\right\}$ by a rounding process. \\
4: Redistribute transmission time to obtain $\left\{t_{i j}\right\}$ and calcu- \\
late $\left\{b_{i}\right\}$.
\end{tabular}

Since NLP is NP-hard, we propose an approximation algorithm termed NLAO-PF, which stands for Non-Linear Approximation Optimization for Proportional Fairness, to simplify the issue and improve the degree of approximation. The steps of NLAO-PF are outlined in Alg. 1.

The basic idea of NLAO-PF is to relax the binary variable $x_{i j}$ such that each user is allowed to associate with multiple APs within a unit time, i.e., $x_{i j}$ can be fractional. Actually we further relax $x_{i j}$ to the extent that any user is allowed to freely associate with all APs by setting $x_{i j}=1$ at the first step. Under such a relaxed condition, we compute the optimal transmission time $t_{i j}^{\prime}$ by solving a relaxed optimization problem (Section IV-A). Taking $t_{i j}^{\prime}$ as a known parameter, we obtain $x_{i j}^{\prime}$, the fractional user-AP association coefficient, by solving a complemented optimization problem (Section IV-B). A rounding technique is then applied to $x_{i j}^{\prime}$ to obtain an approximate integral solution of $x_{i j}$ (Section IV-C). Based on the newly obtained $x_{i j}$, the transmission time $t_{i j}^{\prime}$ is redistributed for the original NLP problem (Section IV-D).

The relaxation involved in NLAO-PF may result in a large integrality gap [35]. To overcome this problem, we modify the objective function of NLP by adding a compensation function $\mathrm{g}(\mathrm{X}, \mathrm{T})$ in NLAO-PF, which is defined as follows:

Definition 1: The compensation of user $i$ on $A P j$ is defined by $w_{i} x_{i j} t_{i j} \log \left(r_{i j}\right)$ if $r_{i j} \geq 1$; and 0 otherwise. Thus the compensation of user $i$ to all APs can be expressed by $w_{i} \sum_{j=1}^{N} x_{i j} t_{i j} \log \left(r_{i j}\right)$. Therefore, the compensation function $g(X, T)$ can be defined correspondingly as follows:

$$
g(X, T)=\sum_{i=1}^{M} w_{i} \sum_{j=1}^{N} x_{i j} t_{i j} \log \left(r_{i j}\right) .
$$

This compensation function is introduced to improve the lower bound of our algorithm to effectively narrow down the integrality gap caused by relaxation. The steps of NLAO-PF are detailed in the following subsections.

\section{A. Relaxed Optimization Program}

The first step of NLAO-PF is to solve the following relaxed optimization problem, denoted by $\mathbf{r}-\mathbf{N L P}$, to obtain an optimal $\left\{t_{i j}^{\prime}\right\}$.

$$
\begin{aligned}
\max & \sum_{i=1}^{M} w_{i} \log \left(\sum_{j=1}^{N} t_{i j}^{\prime} r_{i j}\right)+\sum_{i=1}^{M} w_{i} \sum_{j=1}^{N} t_{i j}^{\prime} \log \left(r_{i j}\right) \\
\text { s.t. } & \sum_{j=1}^{N} t_{i j}^{\prime} \leq 1,1 \leq i \leq M, \\
& \sum_{i=1}^{M} t_{i j}^{\prime}=1,1 \leq j \leq N, \\
& t_{i j}^{\prime} \in[0,1], 1 \leq i \leq M, 1 \leq j \leq N .
\end{aligned}
$$

Compared with (2), r-NLP replaces $t_{i j}$ by $t_{i j}^{\prime}$, sets $x_{i j}=1$, and includes the compensation function in its objective function. The constraint (4b) indicates that the total transmission time of user $i$ with all APs cannot surpass 1; the constraint (4c) requires that the total transmission time of each AP is 
equal to 1 , which means that all APs are saturated in the unit time; and the constraint (4d) defines the range of the variable $t_{i j}^{\prime}$. Obviously, the optimal solution for $\left\{t_{i j}^{\prime}\right\}$ from (4) can be computed in polynomial time [11].

\section{B. Fractional Association}

After solving r-NLP, we obtain the transmission time $\left\{t_{i j}^{\prime}\right\}$. Now we take $\left\{t_{i j}^{\prime}\right\}$ as a known input, and get the fractional user-AP association $\left\{x_{i j}^{\prime}\right\}$. Because of the requirements for solving convex programs, we change the linear equality constraint of NLP to a linear inequality constraint in the following complemented Non-linear Programming (c-NLP) formulation, which does not change the solution value.

$$
\begin{aligned}
\max & \sum_{i=1}^{M} w_{i} \log \left(\sum_{j=1}^{N} x_{i j}^{\prime} t_{i j}^{\prime} r_{i j}\right)+\sum_{i=1}^{M} w_{i} \sum_{j=1}^{N} x_{i j}^{\prime} t_{i j}^{\prime} \log \left(r_{i j}\right) \\
\text { s.t. } & \sum_{j=1}^{N} x_{i j}^{\prime}>0,1 \leq i \leq M \\
& \sum_{i=1}^{M} x_{i j}^{\prime} t_{i j}^{\prime}=1,1 \leq j \leq N \\
& x_{i j}^{\prime} \geq 0,1 \leq i \leq M, 1 \leq j \leq N
\end{aligned}
$$

The objective function of $\mathbf{c - N L P}$ is designed to approximate the optimal solution to NLP. The constraint (5b) indicates that a user should connect with at least one AP; the constraint (5c) forces the total transmission time of each AP be equal to 1 ; and the constraint (5d) defines the range of $x_{i j}^{\prime}$ for the case of fractional association. Note that here we take $\left\{t_{i j}^{\prime}\right\}$ obtained from (4) as the input to $\mathbf{c - N L P}$ and obtain the optimal association $\left\{x_{i j}^{\prime}\right\}$ for $\mathbf{c}-\mathbf{N L P}$ given $\left\{t_{i j}^{\prime}\right\}$.

We can prove that the gap introduced by our relaxation procedure is bounded. Let $f(X, T)=\sum_{i=1}^{M} w_{i} \log \left(\sum_{j=1}^{N} x_{i j} t_{i j} r_{i j}\right)$, and $h(X, T)=f(X, T)+g(X, T)$. Then the objective functions of r-NLP and c-NLP become $h(X=1, T)$ and $h(X, T)$, respectively. Correspondingly, $h\left(X=1, T^{\prime}\right)$ and $h\left(X^{\prime}, T^{\prime}\right)$ are the solutions obtained from r-NLP and c-NLP, respectively.

Theorem 1: Let $\left(X^{*}, T^{*}\right)$ and $\left(X^{\prime}, T^{\prime}\right)$ be the optimal solutions to NLP and to c-NLP, respectively. Then $f\left(X^{*}, T^{*}\right) \leq$ $h\left(X^{\prime}, T^{\prime}\right) \leq 2 f\left(X^{*}, T^{*}\right)$.

Proof: With $\sum_{i=1}^{M} x_{i j}^{\prime} t_{i j}^{\prime}=1$ (constraint (5c)) and $r_{i j} \geq 1$, $f\left(X^{\prime}, T^{\prime}\right) \geq g\left(X^{\prime}, T^{\prime}\right) \geq 0$. Thus, $h\left(X^{\prime}, T^{\prime}\right) \leq 2 f\left(X^{\prime}, T^{\prime}\right) \leq$ $2 f\left(X^{*}, T^{*}\right)$. Since $\left(X=1, T^{*}\right)$ is a feasible solution of the problem $\mathbf{r - N L P}$ and $\left(X=1, T^{\prime}\right)$ is the optimal solution of r-NLP, we have $f\left(X^{*}, T^{*}\right)=f\left(X=1, T^{*}\right) \leq f(X=$ $\left.1, T^{*}\right)+g\left(X=1, T^{*}\right)=h\left(X=1, T^{*}\right) \leq h\left(X=1, T^{\prime}\right) \leq$ $h\left(X^{\prime}, T^{\prime}\right)$, where the last inequality holds from the fact that $h\left(X=1, T^{\prime}\right)$ is feasible to $\mathbf{c}-\mathbf{N L P}$.

\section{Rounding}

In this step, we use the rounding algorithm proposed in [36] to obtain an integral association matrix $X$. That is, we fix the time allocation $\left\{t_{i j}^{\prime}\right\}$ and replace the fractional association $\left\{x_{i j}^{\prime}\right\}$ by a $0-1$ variable $\left\{x_{i j}\right\}$ that encodes the desired association of users to APs. The rounding process contains two main steps: bipartite graph construction and maximum-profit matching, which are detailed as follows.

First, we construct a bipartite graph $G(x)=(U, V, E)$, where the set $U$ represents the users in the network, and the set $V=\left\{v_{j k}: j=1,2, \ldots, N, k=1,2, \ldots, Q_{j}\right\}$, with $Q_{j}=\left\lceil\sum_{i=1}^{M} x_{i j}^{\prime}\right\rceil$. This implies that each AP may have multiple nodes in $V$. The edges in $G(x)$ are constructed according to the following method. If $Q_{j} \leq 1$, there is only one node $v_{j 1}$ corresponding to AP $j$. For each $x_{i j}^{\prime}>0$, add edge $e\left(u_{i}, v_{j 1}\right)$ and set $x^{\prime}\left(u_{i}, v_{j 1}\right)=x_{i j}^{\prime}$, where $x^{\prime}(e)$ is the fractional association weight of the corresponding user and AP. Otherwise, find the minimum index $i_{k}$ such that $\sum_{i=1}^{i_{k}} x_{i j}^{\prime} \geq k$. For $i=i_{k-1}+1, \ldots, i_{k}-1$ and $x_{i j}^{\prime}>0$, add edgee $\left(u_{i}, v_{j k}\right)$ and set $x^{\prime}\left(u_{i}, v_{j k}\right)=x_{i j}^{\prime}$. For $i=i_{k}$, add edge $e\left(u_{i}, v_{j k}\right)$ and set $x^{\prime}\left(u_{i}, v_{j k}\right)=1-\sum_{i=i_{k-1}+1}^{i_{k}-1} x^{\prime}\left(u_{i}, v_{j k}\right)$. If $\sum_{i=1}^{i_{k}} x_{i j}^{\prime}>k$, add edge $e\left(u_{i}, v_{j(k+1)}\right)$ and set $x^{\prime}\left(u_{i}, v_{j(k+1)}\right)=\sum_{i=1}^{i_{k}} x_{i j}^{\prime}-k$. Obviously, $x^{\prime}(e)$ has the following property:

$$
\sum_{i=i_{k-1}+1}^{i_{k}} x^{\prime}\left(u_{i}, v_{j k}\right)= \begin{cases}=1, & k=1,2, \ldots, Q_{j}-1, \\ \leq 1, & k=Q_{j} .\end{cases}
$$

This implies that the sum of the fractional association weights on each node $v_{j k}$ does not exceed one. The profit of each edge $e\left(u_{i}, v_{j k}\right)$ in $E$ is defined to be $w_{i} \log \left(t_{i j}^{\prime} r_{i j}\right)$.

Second, we find a maximum-profit matching $M(x)$ that matches each user node with an AP node in $G(x)$. For each edge $e\left(u_{i}, v_{j k}\right)$ in $M(x)$, schedule user $i$ to AP $j$ and set $x_{i j}=1$. Set other $x_{i j}$ 's to be 0 . Since the fractional association $\left\{x_{i j}^{\prime}\right\}$ specifies a fractional matching, such a maximal matching does exist and it determines the integral association $\left\{x_{i j}\right\}$. More details can be found in [36].

Note that $\left\{t_{i j}^{\prime}\right\}$ and $\left\{x_{i j}^{\prime}\right\}$ are computed from r-NLP and cNLP, respectively. The rounding scheme constructs an integral assignment $\left\{x_{i j}\right\}$. We denote this solution by $f\left(X^{a}, T^{\prime}\right)$, which is also feasible to NLP. Then we have,

Theorem 2: $f\left(X^{a}, T^{\prime}\right) \geq \frac{1}{2} f\left(X^{*}, T^{*}\right)$.

Proof: Note that $\left\{x_{i j}\right\}$ is obtained by employing the rounding scheme proposed by Shmoys and Tardos in [36], which proves the following property: $f\left(X^{a}, T^{\prime}\right) \geq f\left(X^{\prime}, T^{\prime}\right)$. Thus, $f\left(X^{a}, T^{\prime}\right) \geq f\left(X^{\prime}, T^{\prime}\right) \geq \frac{1}{2}\left[f\left(X^{\prime}, T^{\prime}\right)+g\left(X^{\prime}, T^{\prime}\right)\right]=$ $\frac{1}{2} h\left(X^{\prime}, T^{\prime}\right) \geq \frac{1}{2} f\left(X^{*}, T^{*}\right)$, where the last inequality holds from Theorem 1 .

We use an example to demonstrate the rounding process. Suppose that there exist three users and two APs and that we have obtained the following fractional AP association after the second step of Alg. 1: $x_{11}^{\prime}=1, x_{22}^{\prime}=1, x_{31}^{\prime}=\frac{1}{2}=\frac{1}{2}$. The corresponding bipartite graph is illustrated in Fig. 1, where $x_{i j}^{\prime}=1$ for solid edges, $x_{i j}^{\prime}=\frac{1}{2}$ for dashed edges, and the numbers beside the edges are their profits. More specifically, $Q_{1}=Q_{2}=2$; that is, each $\mathrm{AP}$ has two nodes in the bipartite graph. Then, the maximum-profit matching yields 
$x_{11}=x_{22}=x_{31}=1$ with a total profit of 2.7 , which is larger than the fractional association profit 2.5 .

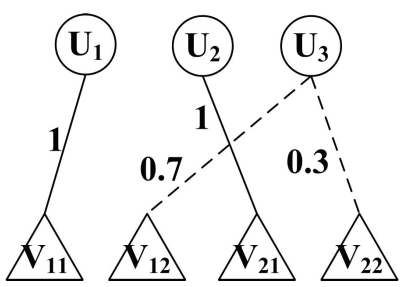

Fig. 1. An Example of Rounding Process

\section{Transmission Time Redistribution}

Since the user-AP association changes after rounding, we need to redistribute the transmission times. This is the last step of NLAO-PF, in which we assign transmission times to users according to proportional fairness.

Theorem 3: Let $\left\{x_{i j}\right\}$ be the integral user-AP association coefficients obtained from the rounding procedure outlined in Section IV-C. Given $\left\{x_{i j}\right\}$, the unique optimal transmission time assigned to user $i$ by AP $j$ according to proportional fairness is

$$
t_{i j}=\frac{w_{i} x_{i j}}{\sum_{k=1}^{M} w_{k} x_{k j}}
$$

Proof: (a) First, we consider the case of a single AP. Assume that the number of users covered by the AP is $m$. Since the objective function of (2) is the sum of logarithms, maximizing the total utility of the user bandwidth (2) is equivalent to maximizing (7):

$$
\prod_{i=1}^{M}\left(t_{i j} r_{i j}\right)^{w_{i}}=\prod_{i=1}^{M}\left(t_{i 1} r_{i 1}\right)^{w_{i}}=\prod_{i=1}^{M}\left(t_{i 1}\right)^{w_{i}} \prod_{i=1}^{M}\left(r_{i 1}\right)^{w_{i}}
$$

Note that $\left\{r_{i 1}\right\}$ is the set of optimization constants. Therefore maximizing (7) is equivalent to maximizing (8):

$\prod_{i=1}^{M}\left(t_{i 1}\right)^{w_{i}}=(\underbrace{t_{11} t_{11} \cdots t_{11}}_{w_{1}})(\underbrace{t_{21} t_{21} \cdots t_{21}}_{w_{2}}) \cdots(\underbrace{t_{M 1} t_{M 1} \cdots t_{M 1}}_{w_{M}})$

Since $\sum_{i=1}^{M} t_{i 1}=1$, (8) is maximized if and only if $\frac{t_{11}}{w_{1}}=$ $\frac{t_{21}}{w_{2}}=\frac{t_{M 1}}{w_{M}}=\frac{1}{\sum_{k=1}^{M} w_{k}}$. Thus we have $t_{i 1}=\frac{w_{i}}{\sum_{k=1}^{M} w_{k}}$.

(b) Now we consider the case of multiple Aे $\overline{\bar{P}}^{1}$. Since $x_{k j}$ is a $0-1$ variable denoting the association coefficient between user $k$ and AP $j, \sum_{k=1}^{M} w_{k} x_{k j}$ is the sum of the weights of all users associated to AP $j$. With a similar analysis as that of case (a), the optimal transmission time given $\left\{x_{i j}\right\}$ can be calculated by (6).

We conclude that given $\left\{x_{i j}\right\}$, our transmission time assignment based on proportional fairness is unique and optimal.

Note that Theorem 3 implies that proportional fairness is equivalent to time-based fairness when all users have the same weight $w_{i}$. This result is consistent with the one proposed in [12] but is obtained from a different angle.
The solution obtained from our algorithm NLAO-PF can be denoted as $f\left(X^{a}, T^{a}\right)$. Based on Theorems 2 and 3, we have $f\left(X^{a}, T^{a}\right) \geq f\left(X^{a}, T^{\prime}\right) \geq \frac{1}{2} f\left(X^{*}, T^{*}\right)$. That is, the approximate solution obtained from NLAO-PF is no less than half of the optimal solution of NLP.

\section{THE BPF ALGORITHM}

In this section, we present a distributed algorithm named Best Performance First (BPF) for AP association to support proportional fairness in dynamic network scenarios.

Note that without collecting the network-wide information a user is only aware of the changes of its currently associated AP, including the join of a new user and the leave of a current user. This implies that it is impossible for a user to switch to an AP whose associated users leave the network. On the other hand, a user's utility can be increased if other users associated with the same AP leave the network, thus this user has no incentive to leave its currently associated AP. As shown in (6), within one unit of transmission time, the portion assigned to each user is proportional to its weight divided by the total weights of all users associated with the same AP. Therefore, re-allocating the optimal transmission time when current users leave from the network on each AP is trivial. Thus in this section, we focus on the case when a user intends to join the network, i.e., how to select the best AP for a new user.

\section{A. BPF Algorighm}

According to IEEE 802.11, by default a new user selects the AP with the strongest received signal strength to associate with once entering a network. However, in some cases, this approach could unfairly overload the APs with the strongest signals and thus reduce the aggregate network throughput. Therefore we hope to seek an AP association method such that the association of a new user results in the most positive impact on the overall network performance. Based on this observation, we construct a performance revenue function, which is defined to be the difference of the bandwidth utilities resulted from the join of the new user to an AP.

We assume that there are $m_{j}$ users associated with AP $j$ before a new user enters $j$ 's coverage area. Denote by $W_{j}$ the sum of the weights of all users on AP $j$. Let $b_{i j}$ denote the bandwidth of user $i$ obtained from AP $j$. According to (6), we have $b_{i j}=\frac{w_{i} r_{i j}}{W_{j}}$, where $W_{j}=\sum_{k=1}^{m_{j}} w_{k}$. Let $r_{0 j}$ be the effective bit rate of the new user, and $w_{0}$ be its weight. If the new user is allowed to join AP $j$, its bandwidth from AP $j$ should become $b_{i j}^{\prime}=\frac{w_{i} r_{i j}}{\left(W_{j}+w_{0}\right)}$. Then the difference of the 
bandwidth utility on AP $j$ can be computed as follows:

$$
\begin{aligned}
\delta_{j} & =\sum_{i=1}^{m_{j}} w_{i} \log \left(b_{i j}^{\prime}\right)+w_{0} \log \left(b_{0 j}^{\prime}\right)-\sum_{i=1}^{m_{j}} w_{i} \log \left(b_{i j}\right) \\
& =\log \left(\prod_{i=1}^{m_{j}}\left(b_{i j}^{\prime}\right)^{w_{i}}\right)+\log \left(b_{0 j}^{\prime}\right)^{w_{0}}-\log \left(\prod_{i=1}^{m_{j}}\left(b_{i j}\right)^{w_{i}}\right) \\
& =\log \left(\prod_{i=1}^{m_{j}}\left(\frac{b_{i j}^{\prime}}{b_{i j}}\right)^{w_{i}}\right)+\log \left(b_{0 j}^{\prime}\right)^{w_{0}} \\
& =\log \left(\prod_{i=1}^{m_{j}}\left(\frac{W_{j}}{W_{j}+w_{0}}\right)^{w_{i}}\right)+\log \left(\frac{r_{0 j} w_{0}}{W_{j}+w_{0}}\right)^{w_{0}} \\
& =\log \left(\frac{W_{j}}{W_{j}+w_{0}}\right)^{W_{j}}+\log \left(\frac{r_{0 j} w_{0}}{W_{j}+w_{0}}\right)^{w_{0}} \\
& =\log \left(\left(\frac{r_{0 j} w_{0}}{W_{j}+w_{0}}\right)^{w_{0}}\left(\frac{W_{j}}{W_{j}+w_{0}}\right)^{W_{j}}\right) .
\end{aligned}
$$

Note that $\delta_{j}$ reflects the improvement of the network performance in terms of bandwidth utility defined in NLAOPF when the new user joins the basic service set of AP $j$. Therefore intuitively the new user should choose the AP with the highest $\delta$ value instead of the one with the strongest signal strength. This idea is summarized by the Best Performance First (BPF) algorithm outlined in Alg. 2, which adopts $\delta$ as the selection criteria for a new user to choose an AP for association.

Also note that the computation of $\delta_{j}$ is based on (6), which computes the optimal transmission time given a userAP association to achieve proportional fairness in NLAO-PF. Considering the fact that BPF assigns the AP to a new user that can achieve the best bandwidth utility improvement while NLAO-PF intends to find the AP association and transmission time assignment such that the total bandwidth utilities can be maximized, we claim that BPF and NLAO-PF employ the same fundamental theory to achieve proportionally fair AP association.

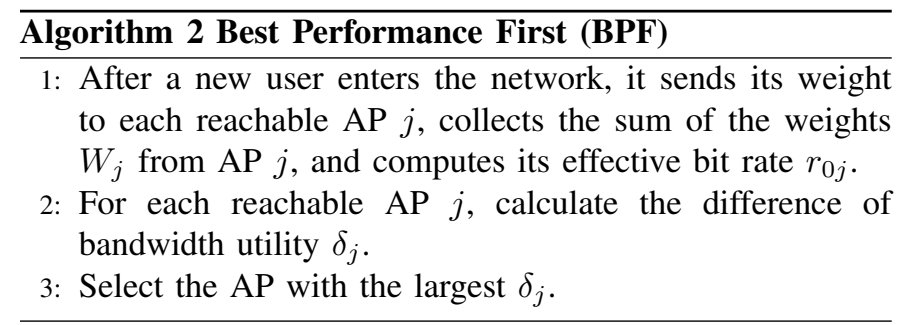

\section{B. Theoretical Analysis}

Eq. (6) indicates that the transmission time of a user is a function of the weights of all users associated with the same AP. Users always intend to obtain a higher bit rate and a larger transmission time to improve their own bandwidth. However, simply selecting the AP with the highest signal strength ignores the transmission time, which may make the network performance declined due to the decreased transmission time of other users associated with the same AP. The bandwidth utility difference defined by (9) considers both the effective bit rate and the weight, which reflects the received signal strength and transmission time, respectively, and therefore providing a better AP selection criterion to achieve proportional fairness.

Furthermore, according to (9), the total network utility increases $\left(\delta_{j}>0\right)$ after accepting a new user only if the following condition is satisfied, i.e.,

$$
\left(r_{0 j} w_{0}\right)^{w_{0}}>\frac{\left(W_{j}+w_{0}\right)^{W_{j}+w_{0}}}{W_{j}^{W_{j}}} .
$$

Let $\alpha_{j}$ be the ratio of $W_{j}$ to $w_{0}$, i.e., $\alpha_{j}=\frac{W_{j}}{w_{0}}$. Note that $\alpha_{j}=0$ if and only if AP $j$ does not have any user associated with it before the new user comes, which is a trivial case. We can express the relationship between the user bit rate and the network utility by Theorem 4 .

Theorem 4: If a new user is associated with AP $j$, the total network utility increases when the new user's bit rate $r_{0 j}$ is larger than a threshold value $\theta_{j}$,

$$
\theta_{j}= \begin{cases}1, & W_{j}=0 \\ \left(1+\alpha_{j}\right)\left(1+\frac{1}{\alpha_{j}}\right)^{\alpha_{j}}, & W_{j}>0\end{cases}
$$

Proof: If $W_{j}=0$, AP $j$ has no user associated with it. According to the property of the logarithm function, the network utility increases only if $r_{0 j}>1$, i.e., $\theta_{j}=1$.

If $W_{j}>0$, there are certain users on AP $j$. Then we have,

$$
\begin{aligned}
\frac{\left(W_{j}+w_{0}\right)^{W_{j}+w_{0}}}{W_{j}^{W_{j}} w_{0}^{w_{0}}} & =\frac{\left(W_{j}+w_{0}\right)^{W_{j}}}{W_{j}^{W_{j}}} \times \frac{\left(W_{j}+w_{0}\right)^{w_{0}}}{w_{0}^{w_{0}}} \\
& =\left(1+\frac{w_{0}}{W_{j}}\right)^{W_{j}}\left(1+\frac{W_{j}}{w_{0}}\right)^{w_{0}}
\end{aligned}
$$

In order to make (10) hold, $r_{0 j}^{w_{0}}>\left(1+\frac{w_{0}}{W_{j}}\right)^{W_{j}}\left(1+\frac{W_{j}}{w_{0}}\right)^{w_{0}}$. Thus we have,

$$
r_{0 j}>\left(1+\frac{w_{0}}{W_{j}}\right)^{\frac{W_{j}}{w_{0}}}\left(1+\frac{W_{j}}{w_{0}}\right)=\left(1+\alpha_{j}\right)\left(1+\frac{1}{\alpha_{j}}\right)^{\alpha_{j}} .
$$

Fig.2 illustrates the impact of different AP selection algorithms on the network performance. A1 and A2 are APs with the same coverage area denoted by circular regions. We assume that users U1, U2 and U3 have the same weight. The solid line labels the AP association for current users, and the dashed lines indicate the possible AP associations for the new user U3. The number beside each line is the effective bit rate if the user is associated with the corresponding AP. Before U3 joins the network, the total throughput is 30 and the network utility is 4.4 according to proportional fairness (each user is allocated half of the transmission time based on (6)). When U3 comes, our BPF algorithm selects A2 for U3, which achieves a total network throughput of 54 and a network utility of 7.57. If the strongest signal first policy (the default user-AP association method in 802.11) is adopted, A1 is selected for $\mathrm{U} 3$, yielding a total throughput of 32 and a network utility of 6.07, which are far less than those obtained from BPF.

On the other hand, the threshold values for U3 to associate with $\mathrm{A} 1$ and $\mathrm{A} 2$ are $\theta_{1}=6.75$ and $\theta_{2}=1$, respectively. This indicates that the total network utility could be increased if the bit rate between $\mathrm{U} 3$ and $\mathrm{A} 1$ is larger than 6.75 , or if the bit rate between $\mathrm{U} 3$ and $\mathrm{A} 2$ is larger than 1; otherwise, the 


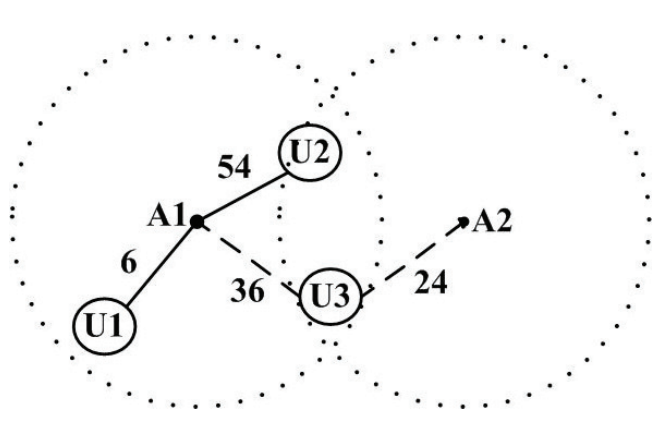

Fig. 2. The AP Association of The New User U3

total utility would decrease after U3 joins the network. For example, if the bit rate between $\mathrm{U} 3$ and A1 (A2) is $6(0.5)$, the new network throughput becomes 22 (30.5) and the utility is reduced from 4.4 to 4.2 (3.7) if U3 is associated with A1 (A2). For such a case, the network utility decreases, resulting in a lower degree of fairness in bandwidth allocation.

\section{Evaluation}

In this section, we report our simulation results for the scenarios where the network contains either static or mobile users. We also compare the performance of our algorithms (the proportional fairness algorithm NLAO-PF and the AP selection algorithm BPF proposed in this paper) with those of the following ones:

- cvapPF: a time-based proportional fairness algorithm proposed in [11]. This algorithm is selected for comparison because it targets the same non-linear problem formulation as that of NLAO-PF but adopts a different relaxation and rounding procedures.

- Strongest Signal First (SSF): the default user-AP association mechanism in the 802.11 standard.

- Norm Load-based Best AP Selection (NLB): an online distributed max-min fairness algorithm designed by $\mathrm{Xu}$ et al. [26]. By comparing with this scheme, the difference between max-min fairness and proportional fairness in multi-rate WLANs can be well demonstrated.

All these algorithms are examined carefully according to the following performance metrics:

- Per-user throughput in Mbps and the corresponding statistical information.

- Jain's fairness index [30], which is defined to be

$$
J=\frac{\left(\sum_{i=1}^{M} b_{i}\right)^{2}}{M\left(\sum_{i=1}^{M} b_{i}^{2}\right)},
$$

where $b_{i}$ is the effective bandwidth allocated to user $i$. Note that a larger value of $J \in[0,1]$ indicates a better fairness.

For ease of comparison, we employ the same simulation settings as those in [11], which are detailed as follows.

Our network contains a total of 20 APs placed on a 5 by 4 grid, with each on a grid point. The coverage area of an AP is set to 150 meters and the distance between two adjacent APs is 100 meters. Assume that the transmission power of an AP is 20 $\mathrm{dBm}$ [37]. There are $50 \sim 300$ users residing in the network, resulting in different levels of network loads. For simplicity, we assume that all users have the same weight. Two types of user distributions are considered: (1) users are randomly and uniformly distributed within the coverage area of the network; (2) users are randomly positioned in a circle-shaped hotspot area with a radius of 100 meters near the center of the 20-AP network. The former simulates the scenario with a balanced user distribution, while the latter simulates the scenario where users are distributed in a particular focused area.

We employ a simple wireless channel model in which the user bit rate only depends on the experienced SINR. The values commonly advertised by $802.11 \mathrm{a} / \mathrm{g}$ are employed in our simulation. Therefore we assume that the bit rate of the users is determined according to Table II. The link gains are modeled by the following equation,

$$
g_{i j}=s_{i j} d_{i j}^{-4}
$$

where $s_{i j}$ is a log-normally distributed shadowing factor, and $d_{i j}$ is the distance between user $i$ and AP $j$. Shadowing factors are generated according to the Viterbi model [38], with $E\left(s_{i j}\right)=0 \mathrm{~dB}$ and $\sigma\left(s_{i j}\right)=10 \mathrm{~dB}$.

We use OMNetpp as the simulator with the corresponding parameters shown in TABLE III.

TABLE III

SiMULATION PARAMATERS

\begin{tabular}{|l|l|}
\hline \multicolumn{1}{|c|}{ Parameter } & \multicolumn{1}{c|}{ Value } \\
\hline Simulation Time & $300 \mathrm{~s}$ \\
\hline Packet Size & $512 \mathrm{bits}$ \\
\hline Transmit Power & $100 \mathrm{~mW}$ \\
\hline SINR Threshold & $6 \mathrm{~dB}$ \\
\hline Noise & $-80 \mathrm{~dB}$ \\
\hline
\end{tabular}

\section{A. Static Network Scenario}

We first report our simulation results for the static network scenario. Assume that all users stay in the network at fixed positions during the whole simulation time.

It is not surprising to observe that by varying the number of users we obtain quantitatively similar results. Therefore in this subsection we only report the results for the 200-user case. The statistics of the achieved throughput and the Jain's fairness index of different algorithms are presented in Table IV. In the following, we give a detailed analysis on the results obtained from different scenarios.

First we compare the two centralized global optimization algorithms: NLAO-PF and cvapPF. Since the problem of AP association based on proportional fairness is NP-hard, these two algorithms can only obtain approximate solutions. For comparison purpose, we use the numerical result obtained from r-NLP without the compensation function $g(X, T)$ as a benchmark and call it FraOp.

Fig. 3 plots the achieved per-user throughput in Mbps vs. user index, with the users sorted by their throughputs in a nondecreasing order. In Fig. 3(a), we observe that in the two user distribution cases, NLAO-PF and cvapPF can both achieve proportional fairness, but NLAO-PF outperforms cvapPF in 
TABLE IV

The Statistics of the Results in Static Network SCEnario (200 users)

\begin{tabular}{|c|c|c|c|c|c|c|}
\hline Case & Algorithm & Max. (Mbps) & Min. (Mbps) & Mean. (Mbps) & Std. (Mbps) & Jain's Fairness Index \\
\hline \multirow{6}{*}{ Uniform } & FraOp & 9.01 & 2.21 & 5.02 & 1.87 & 0.86 \\
\hline & NLAO-PF & 8.75 & 2.21 & 4.99 & 1.88 & 0.85 \\
\hline & BPF & 8.99 & 1.08 & 4.51 & 1.92 & 0.84 \\
\hline & cvapPF & 8.58 & 1.19 & 4.20 & 1.87 & 0.86 \\
\hline & SSF & 9.95 & 0.81 & 4.53 & 2.62 & 0.67 \\
\hline & NLB & 6.65 & 2.12 & 4.16 & 1.57 & 0.90 \\
\hline \multirow{6}{*}{ Hotspot } & FraOp & 8.11 & 2.26 & 4.38 & 1.45 & 0.94 \\
\hline & NLAO-PF & 8.05 & 1.92 & 4.24 & 1.47 & 0.94 \\
\hline & BPF & 7.92 & 1.32 & 3.89 & 1.54 & 0.91 \\
\hline & cvapPF & 7.49 & 1.24 & 3.14 & 1.50 & 0.91 \\
\hline & SSF & 17.99 & 0.96 & 2.14 & 2.25 & 0.70 \\
\hline & NLB & 5.01 & 1.86 & 3.03 & 1.26 & 0.95 \\
\hline
\end{tabular}

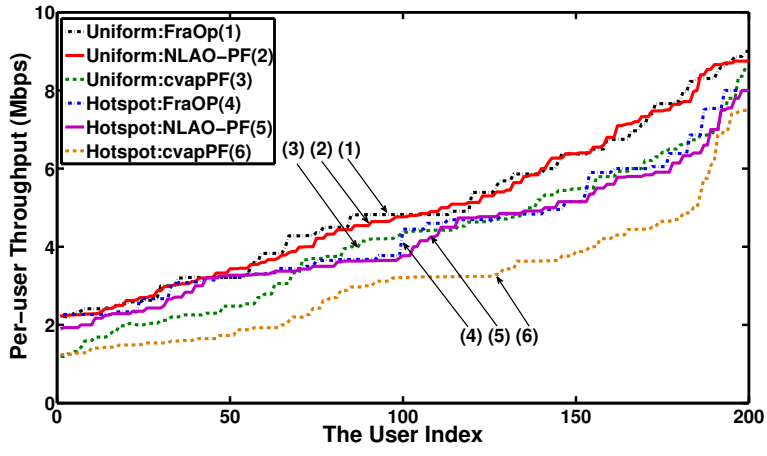

(a) Per-User Throughput of Centralized Algorithms.

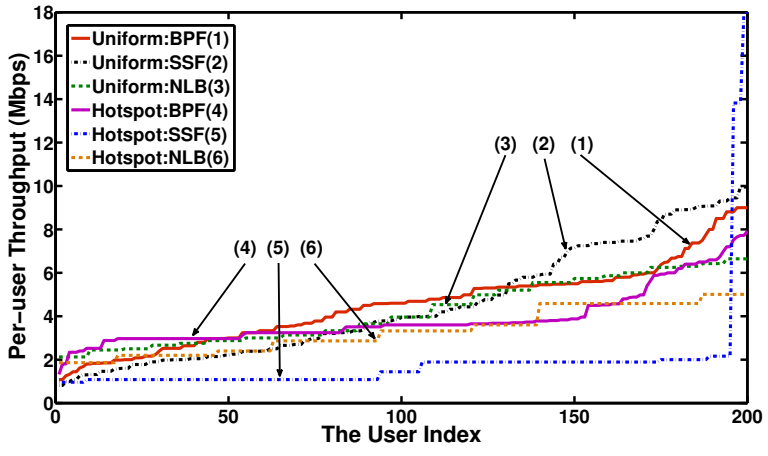

(b) Per-User Throughput of Distributed Algorithms.

Fig. 3. Per-User Throughput of Different Algorithms.

terms of throughput with a value closer to FraOp. As shown in Table IV, the improvements of NLAO-PF over cvapPF in terms of average throughput are $18.8 \%$ and $35.0 \%$ for the cases of users being uniformly distributed in the whole network and in a hotspot area, respectively. On the other hand, the fairness index of these two algorithms is almost the same. Moreover, in both cases, the average throughputs of NLAO$\mathrm{PF}$ are $99.4 \%$ and $96.8 \%$, while those of cvapPF are $83.7 \%$ and $71.7 \%$, normalized over FraOp. Therefore, we conclude that NLAO-PF outperforms cvapPF.

Second, we compare BPF with two other distributed AP association heuristics: SSF and NLB. Fig. 3(b) plots the achieved per-user throughput in Mbps vs. user index, with the users sorted by their throughputs in a non-decreasing order. We observe that when users are randomly and uniformly distributed in the whole network (Fig.3(b) (curves (1) - (3))), the average throughput of SSF is a little bit higher than those of BPF and NLB. However, SSF demonstrates a much larger variance in the user throughput and its fairness is poorer (see Table IV). Nevertheless, the advantages of BPF and NLB cannot be easily manifested under this user distribution scenario because the traffic load is more balanced.

The situation changes in the hotspot case as shown in Fig. 3(b) (curves (4) - (6)). In this case, users reside in the vicinity of certain APs, leading to a more intensive competition for resources and a more imbalanced network load. Obviously, SSF aggravates the extent of load imbalance and enlarges the user throughput variances without considering fairness.
Thus, by taking into account the traffic loads of APs and the achievable transmission rates, both BPF and NLB can significantly improve the user throughput and fairness. More specifically, the average throughput improvement of BPF is $81.8 \%$ compared with SSF, and $28.4 \%$ compared with NLB.

When comparing from the viewpoint of fairness criteria, it can be seen that although max-min fairness (NLB) can obtain a higher fairness index value, it reduces the network throughput. This result confirms the fact that proportional fairness (BPF) provides a more effective tradeoff between fairness and network throughput in a multi-rate WLAN.

\section{B. Dynamic Network Scenario}

In this subsection, we report the simulation results of the three distributed algorithms, namely BPF, SSF, and NLB, for the dynamic network scenario where users are mobile.

We consider the case when users join the network one by one. After a user enters the network, it starts to move according to the following Random Waypoint Mobility Model [39], [40]: the user moves at a random speed to a random waypoint that is uniformly chosen from the given area and pauses at each waypoint for a random interval ranging from 0 to 30 seconds. The random speed is uniformly selected from the range $[0,15$ meters per second].

Fig. 4 reports the aggregated throughput in Mbps vs. the number of users that have joined the network. From the curves (1) - (3) in Fig.4, we observe that SSF obtains a slightly higher aggregated throughput when users are uniformly distributed 
TABLE V

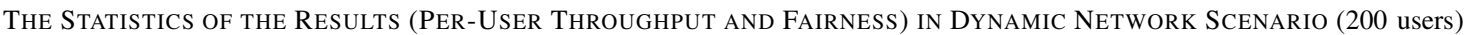

\begin{tabular}{|c|c|c|c|c|c|c|}
\hline Case & Algorithm & Max. (Mbps) & Min. (Mbps) & Mean. (Mbps) & Std. (Mbps) & Jain's Fairness Index \\
\hline \multirow{3}{*}{ Uniform } & BPF & 7.59 & $\mathbf{3 . 8 9}$ & 6.27 & 1.24 & 0.96 \\
\hline & SSF & 7.69 & 3.34 & 6.20 & 1.52 & 0.91 \\
\hline & NLB & 7.32 & 4.47 & 6.21 & 0.96 & 0.98 \\
\hline \multirow{3}{*}{ Hotspot } & BPF & 6.29 & 3.97 & 5.48 & 0.99 & 0.98 \\
\hline & SSF & 6.51 & 1.07 & 4.09 & 2.19 & 0.70 \\
\hline & NLB & 6.02 & 3.34 & 4.63 & 0.81 & 0.99 \\
\hline
\end{tabular}

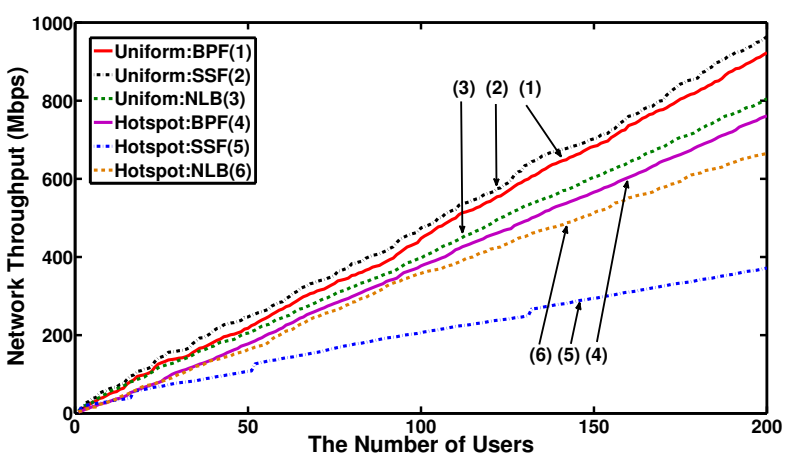

Fig. 4. The Aggregated Throughput vs. the Number of Users.

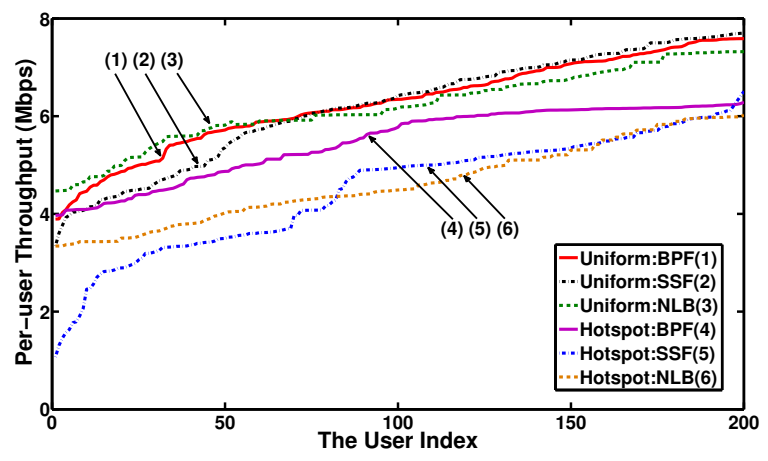

Fig. 5. Per-user Throughput of the Distributed Algorithms in the Dynamic Network Scenario.

in the whole network. This is attributed to the more balanced network load, for which case the advantages of BPF and NLB cannot be easily manifested.

For the hotspot case shown in Fig. 4 (curves (4) - (6)), fairness becomes a key factor because of the load imbalance. Thus, BPF and NLB outperform SSF. On the other hand, it is difficult for NLB to effectively enhance the aggregated throughput since it takes into account the max-min fairness and ignores the network efficiency. Therefore we conclude that $\mathrm{BPF}$ provides a more effective tradeoff between the aggregated throughput and fairness.

The impact of user mobility on the network performance is presented in Fig. 5 and TABLE V. Fig. 5 plots the achieved per-user throughput in Mbps vs. user index, with the users sorted by their throughputs in a non-decreasing order. Although the average throughput of SSF is almost the same as that of BPF for the uniform case, its fairness (Jain's index) is the lowest. In particular, its performance is the worst for the hotspot case in terms of per-user throughput and fairness because it does not consider load balancing in the network. Since NLB aims at maximizing the minimum user throughput and guarantees that all users on the same AP obtain equal throughput, it cannot significantly enhance the network throughput. On the other hand, BPF associates users to APs according to proportional fairness, thus achieving an effective trade-off between throughput and fairness. The statistics of per-user throughput for the dynamic network scenario (TABLE $\mathrm{V})$ indicate that the improvement of the average per-user throughput of BPF is $34.0 \%$ compared with that of SSF, and $18.4 \%$ compared with that of NLB in the hotspot case. Thus, we conclude that BPF performs better than SSF and NLB in terms of per-user throughput.

TABLE VI

THE Number of AP RE-ASSOCIATIONS IN DYNAMIC NETwork SCENARIO (200 users)

\begin{tabular}{|c|c|c|c|c|}
\hline Case & Algorithm & Max. Times & Min. Times & Mean. Times \\
\hline \multirow{3}{*}{ Uniform } & BPF & $\mathbf{1 4}$ & $\mathbf{8}$ & $\mathbf{1 1}$ \\
& SSF & 18 & 10 & 16 \\
& NLB & 14 & 9 & 12 \\
\hline \multirow{3}{*}{ Hotspot } & BPF & $\mathbf{1 0}$ & $\mathbf{7}$ & $\mathbf{8}$ \\
& SSF & 16 & 9 & 12 \\
& NLB & 12 & 7 & 10 \\
\hline
\end{tabular}

The numbers of AP re-associations due to user mobility for the three distributed algorithms are listed in TABLE VI. Note that each re-association involves a de-association from the previous AP. In BPF and NLB, a user performs de-association and then re-association to a different AP by considering both the load of the AP and the data rate, while SSF takes into account the value of SINR only. As a result, BPF and NLB conduct smaller numbers of de-associations/re-associations compared to SSF. Note that more AP re-associations implies less time for user transmissions, leading to a shorter effective transmission time and a lower throughput. Thus with the smallest number of AP re-associations compared to SSF and NLB, BPF can effectively improve the user throughput. Moreover, the least number of AP switching operations also implies that BPF has the highest degree of stability compared to SSF and LLF, which is important as typically users prefer networks providing stable communications.

\section{CONCLUSION}

The widespread of multi-rate WLAN applications makes the network management more complex and critical. Fairness and AP association are two hot issues. In multi-rate WLANs, some users may get starved if fairness is not carefully considered. In this paper, we investigate how to optimize user- 
AP association to achieve proportional fairness. We propose two AP association algorithms, namely NLAO-PF and BPF. Although the problem of optimizing user-AP association to achieve proportional fairness is NP-hard, NLAO-PF obtains a result that is guaranteed to be at least half of the optimal via a compensation function. On the other hand, BPF provides a new AP selection criterion based on a performance revenue function obtained when new users join the network. Simulations confirm that our schemes can achieve proportional fairness in bandwidth allocation and effectively enhance the aggregated throughput. Moreover, our algorithms achieve even better performance when users are distributed in a hotspot area with imbalanced network load.

In our future research, we will consider a more general network model that contains both the uplink and the downlink traffic; moreover, we will investigate the impact of interference on proportional fairness in multi-rate WLANs.

\section{REFERENCES}

[1] G. Tan and J. Guttag, "Time-based fairness improves performance in multi-rate WLANs," in USENIX Annual Technical Conference, June 2004.

[2] M. Heusse, F. Rousseau, G. Berger-Sabbatel, and A. Duda, "Performance anomaly of 802.11b," in IEEE INFOCOM, April 2003, pp. 836843.

[3] A. V. Babu and L. Jacob, "Performance analysis of IEEE 802.11 multirate WLANs: Time based fairness vs throughput based fairness," in Wireless Networks, Communications and Mobile Computing, June 2005, pp. 203-208.

[4] D. Bertsekas and R. Gallager, Data Networks. Prentice-Hall, 1987.

[5] Y. Bejerano and R. S. Bhatia, "MiFi: A framework for fairness and QoS assurance in current IEEE 802.11 networks with multiple access points," in IEEE INFOCOM, March 2004.

[6] Y. Bejerano, S.-J. Han, and L. E. Li, "Fairness and load balancing in wireless LANs using association control," IEEE/ACM Transactions on Networking, vol. 15, no. 3, pp. 560-573, June 2007.

[7] Y. Bejerano and S.-J. Han, "Cell breathing techniques for load balancing in wireless LANs," IEEE Transactions on Mobile Computing, vol. 8, no. 6, pp. 735-749, June 2009.

[8] F. P. Kelly, "Charging and rate control for elastic traffic," European Transaction on Telecommunications, vol. 8, no. 1, pp. 33-37, 1997.

[9] D. Zheng and J. Zhang, "Channel-aware weighted proportional fair medium access control in wireless lans with MIMO links," in QShine, August 2005.

[10] J. Zhang, "Cross-layer rate control in multi-hop networks: Fairness,noisy feedback and stochastic stability," in IEEE Communication Theory Workshop, May 2006.

[11] L. E. Li, M. Pal, and Y. R. Yang, "Proportional fairness in multi-rate wireless LANs," in IEEE INFOCOM, April 2008, pp. 1004-1012.

[12] Y. Le, L. Ma, W. Cheng, X. Cheng, and B. Chen, "Maximizing throughput when achieving time fairness in multi-rate wireless lans," The George Washington University, Tech. Rep. Technical Reports TRGWU-CS-2012-003, June 2012.

[13] T. Bu, L. E. Li, and R. Ramjee, "Generalized proportional fair scheduling in third generation wireless data network," in IEEE INFOCOM, April 2006, pp. 1-12.

[14] Y. Jian and S. Chen, "Can CSMA/CA networks be made fair?" in ACM MobiCom, September 2008, pp. 235-246.

[15] M. Bredel and M. Fidler, "Understanding fairness and its impact on quality of service in IEEE 802.11," in IEEE INFOCOM, April 2009, pp. 1098-1106.

[16] M. Nassiri, M. Heusse, and A. Duda, "A novel access method for supporting absolute and proportional priorities in 802.11 WLANs," in IEEE INFOCOM, April 2008, pp. 709-717.
[17] B. Sadeghi, V. Kanodia, A. Sabharwal, and E. W. Knightly, "OAR: An opportunistic auto-rate media access protocol for ad hoc network," Wireless Networks, vol. 11, no. 1-2, pp. 39-53, January 2005.

[18] A. Banchs, P. Serrano, and H. Oliver, "Proportional fair throughput allocation in multirate IEEE 802.11e wireless LANs," Wireless Networks, vol. 13, no. 5, pp. 649-662, October 2007.

[19] Y. Le, L. Ma, W. Cheng, X. Cheng, and B. Chen, "Maximizing throughput when achieving time fairness in multi-rate wireless lans," in IEEE INFOCOM Mini Conference, March 2012, pp. 3193-3197.

[20] O. Ekici and A. Yongacoglu, "A novel association algorithm for congestion relief in IEEE 802.11 WLANs," in ACM IWCMC, July 2006 , pp. 725-730.

[21] M. Abusubaih and A. Wolisz, "An optimal station association policy for multi-rate IEEE 802.11 wireless LANs," in ACM MSWiM, October 2007, pp. 117-123.

[22] L.-H. Yen, J.-J. Li, and C.-M. Lin, "Stability and fairness of ap selection games in ieee 802.11 access networks," IEEE Transactions on Vehicular Technology, vol. 60, no. 3, pp. 1150-1160, March 2011.

[23] S. Keranidis, T. Korakis, I. Koutsopoulos, and L. Tassiulas, "Contention and traffic load-aware association in ieee 802.11 wlans: Algorithms and implementation," in IEEE WiOpt, May 2011, pp. 334-341.

[24] H. Gong, K. Nahm, and J. Kim, "Access point selection tradeoff for IEEE 802.11 wireless mesh network," in IEEE CCNC, January 2007, pp. $818-822$.

[25] S. K. Dandapat, B. Mitra, R. R. Choudhury, and N. Ganguly, "Smart association control in wireless mobile environment using max-flow," IEEE Transactions on Network and Service Management, vol. 9, no. 1, pp. 73-86, March 2012.

[26] F. Xu, C. C. Tan, Q. Li, G. Yan, and J. Wu, "Designing a practical access point association protocol," in INFOCOM, March 2010, pp. 1-9.

[27] I. Koukoutsidis and V. A. Siris, "Access point assignment algorithms in WLANs based on throughput objective," in ICST WiOPT, March 2008 , pp. 375-383.

[28] L. Xie, Q. Li, W. Mao, J. Wu, and D. Chen, "Achieving efficiency and fairness for association control in vehicular networks," in IEEE ICNP, October 2009, pp. 324-333.

[29] W. Li, Y. Cui, and X. Cheng, "Achieving proportional fairness via ap power control in multi-rate WLANs," IEEE Transactions on Wireless Communications, vol. 10, no. 11, pp. 3784-3792, November 2011.

[30] R. Jain, D.-M. Chiu, and W. R. Hawe, "A quantitative measure of fairness and discrimination for resource allocation in shared computer system," Digital Equipment, Tech. Rep. DEC-TR-301, Tech. Rep., September 1984.

[31] A. Akella, G. Judd, S. Seshan, and P. Steenkiste, "Self-management in chaotic wireless deployments," in ACM MobiCom, August 2005, pp. 185-199.

[32] B. Kauffmann, F. Baccelli, A. Chaintreau, V. Mhatre, K. Papagiannaki, and C. Diot, "Measurement-based self organization of interfering 802.11 wireless access networks," in IEEE INFOCOM, May 2007, pp. 14511459.

[33] "IEEE Std 802.11a high-speed physical layer in the $5 \mathrm{GHz}$ band," IEEE Standard 802.11a, 1999.

[34] "IEEE Std 802.11g further higher data rate extension in the $2.4 \mathrm{GHz}$ band," IEEE Standard $802.11 \mathrm{~g}, 2003$.

[35] Y. Azarm and A. Epstein, "Convex programming for scheduling unrelated parallel machines," in ACM symposium on Theory of computing, May 2005, pp. 331-337.

[36] D. B. Shmoys and E. Tardos, "An approximation algorithm for the generalized assignment problem," Mathematical Programming, vol. 62, no. 3, pp. 461-474, December 1993.

[37] "IEEE Std $802.11 \mathrm{k}$ radio resource measurement of wireless LANs," IEEE Standard 802.11k, 2008.

[38] A. J. Viterbi, CDMA: Principles of Spread Spectrum Communication New York: Addison-Wesley, 1995.

[39] C. Bettstetter, H. Hartenstein, and X. Pérez-Costa, "Stochastic properties of the random waypoint mobility model," ACM Wireless Networks, vol. 10, no. 5, pp. 555-567, September 2004.

[40] A. Jindal and K. Psounis, "Fundamental mobility properties for realistic performance analysis of intermittently connected mobile networks," in IEEE PerCom Workshops, March 2007, pp. 59-64. 


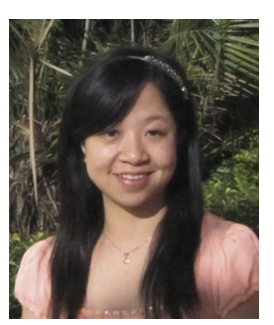

Wei $\mathbf{L i}$ is a second year Ph.D student from the Department of Computer Science, The George Washington University, Washington DC. She received her M.S. degree in Computer Science from Beijing University of Posts and Telecommunications in 2011. She won the sole Best Paper Award in 5th international conference WASA on Aug. 2010. Her current research interests include network resource management, algorithm design and analysis, and cooperative communications in wireless networks. She is a student member of IEEE.



Shengling Wang received the $\mathrm{PhD}$ degree in computer science from Xi'an Jiaotong University, China, in 2008. After that, she worked at Tsinghua University as a postdoctor. Now, she is an associate professor at the Institute of Computing Technology, Chinese Academy of Sciences. Her current research interests include the mobility management, routing and load balancing in wireless and mobile networks.



Yong Cui received the BE degree and the Ph.D degree from Tsinghua University, China in 1999 and 2004, respectively. He is currently an associate professor in Tsinghua University, Council Member in China Communication Standards Association, CoChair of IETF IPv6 Transition WG Software. Having published more than 100 papers in refereed journals and conferences, he received the National Science and Technology Progress Award (Second Class) in 2005, the Influential Invention Award of China Information Industry in 2004, and two best paper awards in ACM ICUIMC 2011 and WASA 2010. Holding more than 40 patents, he is one of the authors in RFC 5747 and RFC 5565 for his proposal on IPv6 transition technologies. His major research interests include mobile wireless Internet and computer network architecture.

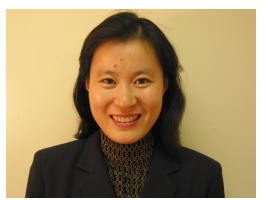

Xiuzhen Cheng received her MS and $\mathrm{PhD}$ degrees in computer science from the University of Minnesota - Twin Cities, in 2000 and 2002, respectively. She is an associate professor at the Department of Computer Science, The George Washington University, Washington DC. Her current research interests focus on cognitive radio networks, mobile handset networking systems (mobile health and safety), wireless and mobile computing, sensor networking, wireless and mobile security, and algorithm design and analysis. She has served on the editorial boards of several technical journals and the technical program committees of various professional conferences/workshops. She also has chaired several international conferences. She worked as a program director for the US National Science Foundation (NSF) from April to October in 2006 (full time), and from April 2008 to May 2010 (part time). She received the NSF CAREER Award in 2004. She is a senior member of IEEE and a member of ACM.

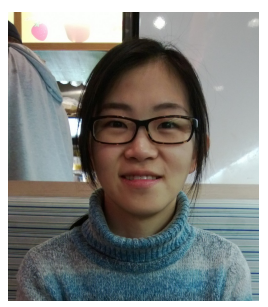

Ran Xin received the B.S. degree in E-commerce Engineering with Laws from Beijing University of Posts and Telecommunications in 2012, and currently pursuing the M.S. degree in Computer Science at Illinois Institute of Technology. Her interest includes wireless networking.
Mznah Al-Rodhaan has received BS in Computer Applications (Hon) and MS in Computer Science both from King Saud University, on 1999 and 2003 respectively. In 2009, she received her Ph.D. in Computer Science from the University of Glasgow, Scotland, UK. She is currently working as an assistant professor and vice chair of the Computer Science department, College of Computer and Information Sciences, King Saud University, Riyadh, Saudi Arabia. Moreover, she served in the editorial board of the Ad Hoc journal (Elsevier) and has participated in several international conferences. Her current research interest includes: Mobile Ad Hoc Networks, Wireless Sensor Networks, Cognitive Networks, Network Security, and High Performance Computing.



Abdullah Al-Dhelaan has received BS in Statistics (Hon) from King Saud University, on 1982, and the MS and Ph.D. in Computer Science from Oregon State University on 1986 and 1989 respectively. $\mathrm{He}$ is currently Associate Professor of Computer Science, Chairman of the join Ph.D. program, and Director General for the Center for International Collaboration and Visiting Professors, College of Computer and Information Sciences, King Saud University, Riyadh, Saudi Arabia. He has guest edited several special issues for the Telecommunication Journal (Springer), and the International Journal for Computers and their applications (ISCA). Moreover, he is currently on the editorial boards of several journals such and Computer Network (Elsevier) and The International Journal of Computers and their applications. His current research interest includes: Mobile Ad Hoc Networks, Sensor Networks, Cognitive Networks, Network Security, and High Performance Computing. 